Check for updates

Cite this: Chem. Sci., 2019, 10, 1219

๑ All publication charges for this article have been paid for by the Royal Society of Chemistry

Received 2nd October 2018

Accepted 9th November 2018

DOI: $10.1039 / \mathrm{c} 8 \mathrm{sc} 04363 a$

rsc.li/chemical-science

\title{
Novel near-infrared II aggregation-induced emission dots for in vivo bioimaging $\dagger$
}

\author{
Jiacheng Lin,,$^{\mathrm{ab}}$ Xiaodong Zeng,,$^{\mathrm{ab}}$ Yuling Xiao,,$^{\mathrm{ab}}$ Lin Tang, ${ }^{a}$ Jinxia Nong, ${ }^{a}$ \\ Yufang Liu, ${ }^{a}$ Hui Zhou, (D) ab Bingbing Ding, ${ }^{a}$ Fuchun $X u,{ }^{c}$ Hanxing Tong, ${ }^{d}$ Zixin Deng ${ }^{a}$ \\ and Xuechuan Hong (D) *abc
}

\begin{abstract}
Near-infrared II fluorescence imaging holds great promise for in vivo imaging and imaging-guided surgery with deep penetration and high spatiotemporal resolution. However, most NIR-II aromatic luminophores suffer from the notorious aggregation-caused quenching (ACQ) effect in the aqueous solution, which largely hinders their biomedical application in vivo. In this study, the first NIR-II organic aggregationinduced emission (AIE) fluorophore (HLZ-BTED), encapsulated as nanoparticles (HLZ-BTED dots) for in vivo biomedical imaging, was designed and synthesized. The NIR-II AIE HLZ-BTED dots showed high temporal resolution, high photostability, outstanding water-solubility and biocompatibility in vitro and in vivo. The HLZ-BTED dots were further used for long-term breast tumor imaging and visualizing tumorfeeding blood vessels, long-term hind limb vasculature and incomplete hind limb ischemia. More importantly, as a proof-of-concept, this is the first time that non-invasive and real-time NIR-II imaging of the gastrointestinal tract in health and disease has been performed, making the AIE dots a promising tool for gastrointestinal (Gl) tract research, such as understanding the healthy status of Gl peristalsis, diagnosing and evaluating intestinal motility dysfunction, and assessing drug effects on intestinal obstruction.
\end{abstract}

\section{Introduction}

Optical fluorescence imaging in the near-infrared window (NIR, $700-1700 \mathrm{~nm}$ ) is a well-established and powerful tool for biomedical application in scientific research and clinical practice, such as noninvasive in vivo vascular imaging, ${ }^{1}$ lymphatic mapping, ${ }^{2}$ cancer diagnosis, ${ }^{3}$ and image-guided surgery. ${ }^{4}$ Compared with the conventional first near-infrared window (NIR-I, 700-900 nm) fluorescence imaging, fluorescence imaging in the second near-infrared window (NIR-II, 1000-1700 $\mathrm{nm}$ ) has deeper penetration and higher spatiotemporal resolution due to low photon absorption and scattering and low auto-fluorescence of normal biological tissues. ${ }^{5}$ Numerous organic or inorganic nanostructured NIR-II contrast agents

${ }^{a}$ State Key Laboratory of Virology, Key Laboratory of Combinatorial Biosynthesis and Drug Discovery (MOE), Hubei Provincial Key Laboratory of Developmentally Originated Disease, Wuhan University School of Pharmaceutical Sciences, Wuhan 430071, China.E-mail: xhy78@whu.edu.cn

${ }^{b}$ Shenzhen Institute of Wuhan University, Shenzhen, 518057, China

'Innovation Center for Traditional Tibetan Medicine Modernization and Quality Control, Medical College, Tibet University, Lhasa, 850000, China

${ }^{d}$ Department of General Surgery, Zhongshan Hospital, Fudan University, Shanghai, 200032, China

† Electronic supplementary information (ESI) available. See DOI: 10.1039/c8sc04363a

\$ These authors contributed equally to this work. such as rare earth nanoparticles (NPs), ${ }^{6}$ quantum dots (QDs), ${ }^{7}$ carbon nanotubes (CNTs), ${ }^{8}$ and conjugated polymer NPs ${ }^{9}$ have been reported for biological imaging. Recently, the first rapidly renally excreted NIR-II fluorophore CH1055 has been developed based on a benzobisthiadiazole (BBTD)-based donor-acceptordonor (D-A-D) structure for high-quality NIR-II biomedical imaging. ${ }^{10}$ After that, a series of small-molecule organic NIR-II dyes were reported for use in bioimaging with or without the BBTD core. ${ }^{11}$ However, most of these NIR-II aromatic luminophores suffer from the notorious aggregation-caused quenching (ACQ) effect, with strong emission as isolated molecules but poor emission efficiency in the aggregate state due to intense intermolecular $\pi-\pi$ stacking interactions, which largely hinders their biomedical application in NIR-II imaging.

Research efforts to solve the ACQ problem by hampering chromophore aggregation have ended with limited success until 2001. ${ }^{12}$ A novel AIE phenomenon, an exactly opposite phenomenon to overcome the notorious ACQ effect was reported by Tang and his co-workers. ${ }^{12}$ The luminescent molecules with AIE characteristics usually show strong fluorescence emission upon aggregation, and weak or non-emissive fluorescence in dilute solvents. So far, a variety of small fluorescent organic molecules have been developed as AIE fluorogens (AIEgens) with visible (Vis) or NIR-I fluorescence emission. ${ }^{13}$ Recently, two short-wave infrared (SWIR, 900-1700 nm) AIEgens TQ-BPN and TB1 were reported with the maximum fluorescence 
emission peak at $810 \mathrm{~nm}$ and $975 \mathrm{~nm}$ for high-resolution bioimaging and dual fluorescence and photoacoustic imaging for precise brain cancer diagnosis. ${ }^{\mathbf{1 4 , 1 5}}$ Hence, many efforts have been made to optimize the brightness of small-molecule NIR-II fluorophores and increase the emission wavelengths of NIR-II AIEgens.

Herein, a novel NIR-II small-molecule organic fluorogen HLZ-BTED with AIE characteristics based on BBTD and tetraphenylethylene (TPE) was designed and facilely prepared. ${ }^{16}$ The fluorescence emission wavelength of encapsulated HLZ-BTED dots was longer than $1000 \mathrm{~nm}$. The HLZ-BTED dots were used for passive targeted long-term NIR-II fluorescence imaging of breast cancer based on the enhanced permeability and retention (EPR) effect, and in vivo visualizing of tumor-feeding arteries, long-term hind limb vasculature and incomplete hind limb ischemia. In a proof-of-concept study, we have developed a new concept for the real-time imaging of normal gastrointestinal motility and intestinal obstruction in a mouse model using the NIR-II HLZ-BTED dots, which could be a powerful biomedical research tool for identifying gastrointestinal function and diseases.

\section{Results and discussion}

A new type of NIR-II AIE dye HLZ-BTED with a different core structure from TB1 was designed and synthesized. Based on the D-A-D scaffold, herein we incorporated a 3,4-ethylenedioxythiophene (EDOT) spacer and 4,4'-(2,2-diphenylethene-1,1-diyl)bis( $N, N$-diethylaniline $)$ as the AIE functional group for the fluorescent compound HLZ-BTED and investigated the relationship between the structure and absorption/ fluorescence properties. DFT calculations with the B3LYP exchange functional employing $6-31 G(d, p)$ basis sets were performed to obtain the highest occupied molecular orbital (HOMO) and lowest unoccupied molecular orbital (LUMO) of TQ-BPN, TB1 and HLZ-BTED. The HOMOs of HLZ-BTED are delocalized on the whole molecular backbone including BBTD, EDOT, and TPE units, while the LUMOs are mainly localized on the electron-deficient BBTD acceptor and the EDOT donor (Fig. 1B). As shown in Fig. S1A and S1B, $\uparrow$ the electronic
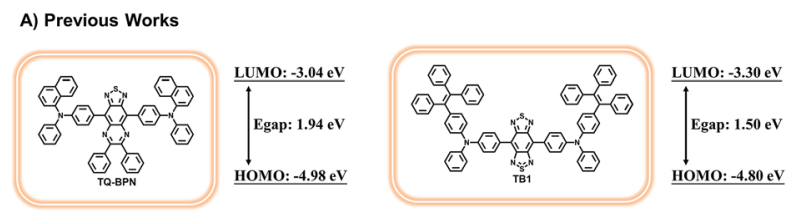

B) This Work

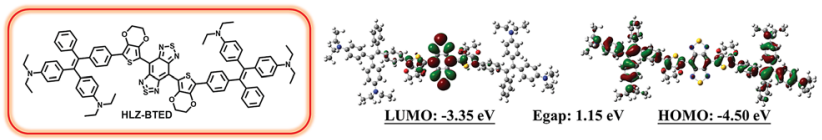

Fig. 1 (A) Chemical structures and electronic properties of previously reported SWIR AIE fluorogens (TQ-BPN and TB1). (B) Chemical structure, frontier molecular orbital amplitude plots and electronic properties of the BBTD-based NIR-II AIE fluorogen. All molecular fluorophores were calculated using B3LYP/6-31G (d, p). structures of TQ-BPN and TB1 are similar to that of the HLZBTED fluorophore. However, the energy bandgaps of HLZ-BTED $(1.15 \mathrm{eV})$ are lower than those of TQ-BPN (1.94 eV) and TB1 (1.50 eV). Thus, HLZ-BTED showed larger red-shifts of the absorption spectra and fluorescence emission peaks compared to TQ-BPN and TB1 (Fig. 1 and S1C $\dagger$ ).

The small-molecule AIE fluorophore, HLZ-BTED, was facilely prepared according to the synthetic route shown in Fig. 2A. 1-(4Bromophenyl)-1-phenyl-2,2-bis(4-diethylamino)phenylethene 2 was prepared via a McMurry coupling reaction between 4-bromobenzophenone and 4,4'-bis(diethylamino)benzophenone using titanium tetrachloride as the catalyst and zinc dust as the reducing agent. ${ }^{17}$ The boric acid ester substrate $\mathbf{3}$ was then obtained from compound 2 in the presence of bis(pinacolato) diboron and $\mathrm{PdCl}_{2}(\mathrm{dppf})$ in dioxane. A Suzuki cross-coupling reaction between compound 4 (ref. 18) and compound 3 using $\mathrm{PdCl}_{2}(\mathrm{dppf}) \mathrm{CH}_{2} \mathrm{Cl}_{2}$ as the catalyst in THF to afford the coupling adduct 5 , followed by zinc reduction and $N$-thionylanilineinduced ring closure resulted in the formation of HLZ-BTED. The structure of HLZ-BTED was characterized using NMR and MALDI-TOF-MS (Fig. S11-S17 $\dagger$ ). The AIE properties of HLZBTED were investigated in THF-water mixtures with changing water volume fractions $\left(f_{\mathrm{w}} \mathrm{s}\right)$. As shown in Fig. 2B, HLZ-BTED exhibited weak NIR-II fluorescence emission in the pure THF solution, but extremely strong fluorescence emission in the THF-water mixed solution with $95 \% f_{\mathrm{w}}$. To further confirm the AIE properties of HLZ-BTED, the fluorescence emission spectra of the fluorophore in THF-water mixtures with changing $f_{\mathrm{w}} \mathrm{S}$ were subsequently collected under $785 \mathrm{~nm}$ excitation (Fig. 2C) and investigated. The fluorescence (FL) intensity of HLZ-BTED significantly decreased with the gradual increase of $f_{\mathrm{w}}$ from 0 to $40 \%$, which was possibly due to the increased solvent polarity. The FL intensity of HLZ-BTED increased sharply on further increasing the $f_{\mathrm{w}}$ from 40 to $90 \%$, indicating a typical AIE characteristic, and remained stable with the increase of $f_{\mathrm{w}}$ from

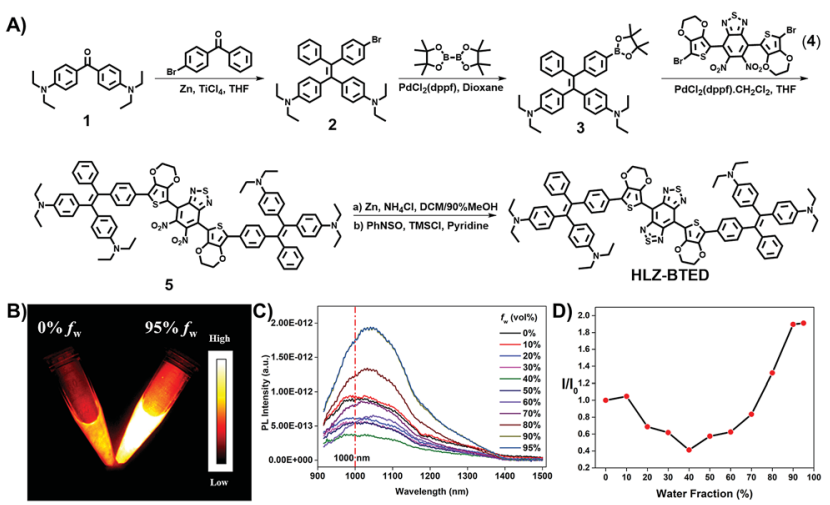

Fig. 2 (A) Synthetic routes of the AIE fluorophore HLZ-BTED based on BBTD and TPE. (B) NIR-II fluorescence images (808 $\mathrm{nm}$ excitation, 1000 LP, $10 \mathrm{~ms}$ ) of HLZ-BTED in pure tetrahydrofuran (THF) and a THF/ water mixture with $95 \%$ water volume fraction $\left(f_{\mathrm{w}}\right)$. The concentration is $2 \times 10^{-5} \mathrm{M}$. (C) Fluorescence (FL) emission spectra of HLZ-BTED in THF/water mixtures with different $f_{\mathrm{w}}$ (0\% to $95 \%$ ). Excitation wavelength at $785 \mathrm{~nm}$. (D) FL intensity ratio (calculated from Fig. $2 \mathrm{C}$ ) $\left(/ / I_{0}\right)$ of the HLZ-BTED fluorophore in different $f_{\mathrm{w}} s$. $I_{0}$ is the FL intensity of HLZBTED in pure THF. 
90 to $95 \%$ (Fig. 2D). This phenomenon may stem from the insolubility of the organic fluorophore in water and results in efficient aggregate emission with a high $f_{\mathrm{w}}$. The molecular fluorophore HLZ-BTED with efficient aggregate emission in water is eligible for further NIR-II biomedical fluorescence imaging.

To illustrate the feasibility of HLZ-BTED for bioimaging application, we fabricated water-soluble and biocompatible AIE dots (HLZ-BTED dots) through a nanoprecipitation method by using 1,2-distearoyl-sn-glycero-3-phosphoethanolamine- $N$ (methoxy(polyethylene glycol)-5000) (DSPE-PEG ${ }_{5000}$ ) as the encapsulation matrix (Fig. 3A). Briefly, a HLZ-BTED/THF mixture was quickly added into the DSPE-PEG ${ }_{5000}$ deionized water solution in an ice bath under continuous sonication. Then, the remaining $\mathrm{THF}$ in the mixture was removed completely under a nitrogen flow. The redundant DSPE-PEG 5000 was removed by ultrafiltration using $50 \mathrm{kDa}$ centrifugal filter devices to obtain HLZ-BTED dots. The synthesized HLZ-BTED dots showed high monodispersity and homogeneity with an average particle size of $\sim 50 \mathrm{~nm}$ as determined by transmission electron microscopy (TEM, Fig. 3B) and a hydrodynamic diameter of $\sim 60 \mathrm{~nm}$ as determined by dynamic light scattering (DLS, Fig. S3A $\dagger$ ). The zeta potential distribution of the HLZBTED dots was measured and the colloidal stability of the AIE dots under physiological conditions was evaluated. The results have demonstrated that the nanoparticles have negative surface

A)

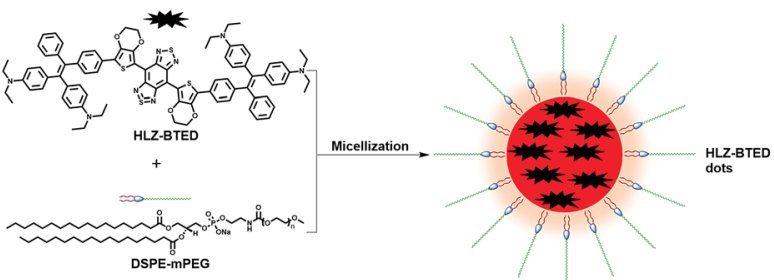

B)
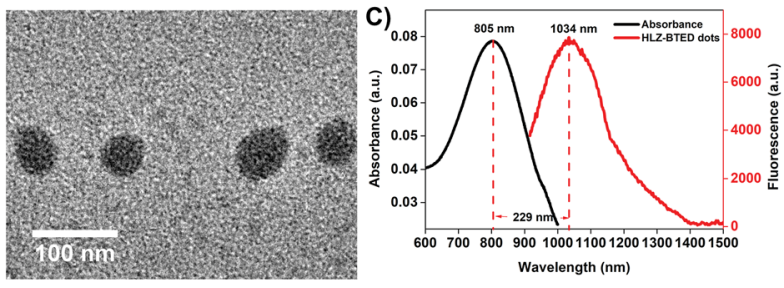

D)
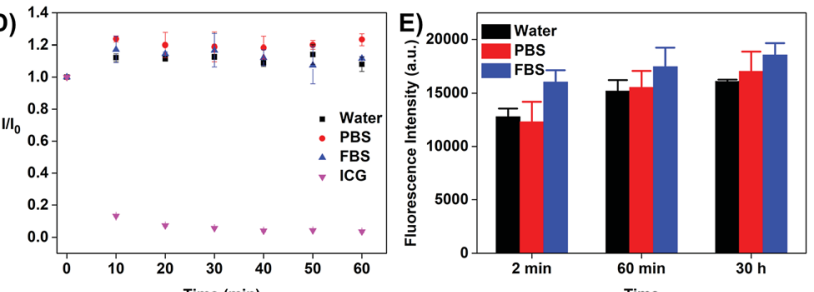

Fig. 3 (A) Schematic illustration of the preparation method of NIR-II AIE nanoparticles (HLZ-BTED dots) via micellization. (B) Representative TEM image of the HLZ-BTED dots. Scale bar: $100 \mathrm{~nm}$. (C) UV-vis-NIR absorption spectrum and NIR-II fluorescence emission spectrum (785 $\mathrm{nm}$ laser excitation) of the HLZ-BTED dots in aqueous solution. (D) Photo-stability test of the AIE HLZ-BTED dots in water, PBS, fetal bovine serum (FBS) and ICG in water under continuous $808 \mathrm{~nm}$ laser $\left(180 \mathrm{~mW} \mathrm{~cm}^{-2}\right)$ irradiation for $60 \mathrm{~min}$. (E) Quantitative analysis of the NIR-II fluorescence intensity of the HLZ-BTED dots in water, PBS, and FBS after 2 min to $30 \mathrm{~h}$ incubation. charges and excellent stability in water and physiological media (Fig. S3B and S4†). The UV-vis-NIR absorption and NIR-II fluorescence emission spectra of the HLZ-BTED dots in water were investigated under $785 \mathrm{~nm}$ excitation. As shown in Fig. 3C, the absorption peak was at $\sim 805 \mathrm{~nm}$, while the fluorescence emission peak was at $\sim 1034 \mathrm{~nm}$ with a $229 \mathrm{~nm}$ Stokes shift. In Fig. S2, $\uparrow$ the NIR-II quantum yield (QY) of the HLZ-BTED dots in water was measured to be $0.18 \%$ under $785 \mathrm{~nm}$ laser excitation using IR-26 dye as a reference $(\mathrm{QY}=0.5 \%)$, which was around two-fold higher than that of the fluorophore in THF $(\mathrm{QY}=0.1 \%)$ and matched well with the results for exploring the AIE property in Fig. 2D. The large Stokes shift and appropriate fluorescence efficiency of the HLZ-BTED dots are remarkably beneficial for NIR-II AIE bioimaging.

The HLZ-BTED dots showed superior photostability in water, phosphate buffered saline (PBS), and fetal bovine serum (FBS) under continuous $808 \mathrm{~nm}$ laser irradiation for $1 \mathrm{~h}$ at a power density of $180 \mathrm{~mW} \mathrm{~cm}^{-2}$, while indocyanine green (ICG), which is approved by the FDA for NIR-I biomedical fluorescence imaging in water, showed a drastic decrease in fluorescence intensity using the same measurement method (Fig. 3D). To explore time-dependent fluorescence stability in different media, the variation in fluorescence intensity of the HLZ-BTED dots in water, PBS, and FBS was observed at different time points ( $2 \mathrm{~min}, 60 \mathrm{~min}$ and $30 \mathrm{~h}$ ) after incubation at room temperature, exhibiting excellent temporal stability without an obvious change in the FL intensity (Fig. 3E). These photo and temporal stability results demonstrated that the HLZ-BTED dots could be useful for long-term in vivo imaging.

To further demonstrate the capability of the HLZ-BTED dots as a NIR-II imaging contrast agent in vivo, the pharmacokinetics and potential toxicity of the HLZ-BTED dots were carefully evaluated. The pharmacokinetics were studied via the measurement of the blood circulation half-life. The half-life time of the HLZ-BTED dots was $204 \mathrm{~min}$ (Fig. S5†). The long circulation time of the AIE dots in blood was mainly due to the strong interaction between the HLZ-BTED dots and serum albumin (Fig. S6, $\uparrow K_{\mathrm{D}}=0.0303 \mathrm{nM}$ ), and allowed them to reach their imaging target. The cytotoxicity of the HLZ-BTED dots to 4T1 mammary cancer and L929 mouse fibroblast cell lines was demonstrated using the standard 3-(4,5-dimethylthiazol-2-yl)2,5-diphenyl tetrazolium bromide (MTT) assay. As shown in Fig. 4A, no obvious cytotoxicity was observed even when the concentration was increased to $150 \mu \mathrm{g} \mathrm{mL} \mathrm{m}^{-1}$. The long-term potential toxicity of the HLZ-BTED dots in normal KM mice was evaluated. A solution of PBS $(0.2 \mathrm{~mL}, n=3)$ and medium dose HLZ-BTED dots $\left(0.2 \mathrm{~mL}, 7.5 \mathrm{mg} \mathrm{kg}^{-1}, n=3\right)$, or high dose HLZBTED dots $\left(0.2 \mathrm{~mL}, 15 \mathrm{mg} \mathrm{kg}^{-1}, n=3\right)$ was injected into normal KM mice by intravenous injection (i.v.) via the tail vein. No significant difference in body weight was observed between the PBS control group and the HLZ-BTED dot administered groups during the research process (31 days) (Fig. 4B). Moreover, the major organs of the mice, including the heart, liver, spleen, lungs and kidneys were obtained and stained with hematoxylin and eosin (H\&E) for histopathological study at 31 days after treatment. The H\&E staining results shown in Fig. 4C exhibited no obvious necrosis or inflammation lesions in all the major 

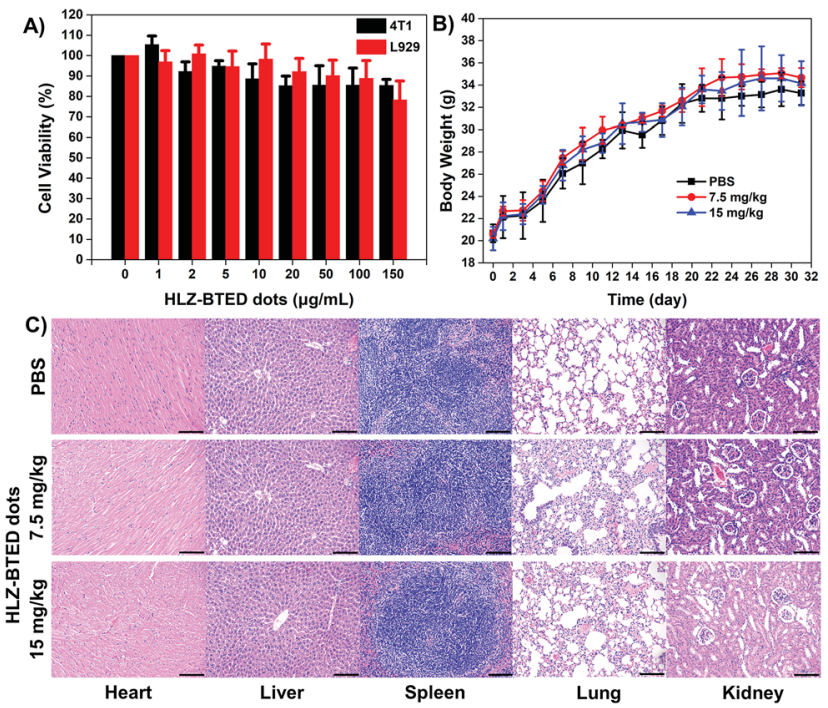

Fig. 4 Biocompatibility study of the HLZ-BTED dots. (A) Cell viability of $4 \mathrm{~T} 1$ and $\mathrm{L} 929$ cells after incubation with different concentrations of the HLZ-BTED dots for $24 \mathrm{~h}(n=3)$. (B) Body weight of normal mice on different days $(n=3)$ treated with PBS, $7.5 \mathrm{mg} \mathrm{kg}^{-1}$, and $15 \mathrm{mg} \mathrm{kg}^{-1}$ HLZ-BTED dots. (C) Representative hematoxylin and eosin stained images of major organs (heart, liver, spleen, lungs, and kidneys) from the control mice and HLZ-BTED dot injected mice at 31 days posttreatment. Scale bar: $100 \mu \mathrm{m}$.

organs, indicating that the HLZ-BTED dots have excellent biocompatibility and are more applicable for NIR-II bioimaging in vivo.

Breast cancer is the most common cancer in women worldwide. ${ }^{19}$ Early diagnosis of breast cancer with the use of systemic therapy has an excellent prognosis and increases the long-term disease-free survival (DFS). ${ }^{20}$ Firstly, the in vitro cellular endocytosis of the HLZ-BTED dots was studied using 4T1 breast cancer cells. As shown in Fig. S7, $\uparrow$ the NIR-II fluorescence intensity of the 4T1 cells incubated with the HLZ-BTED dots showed explicit time dependence, suggesting that the AIE dots can permeate into the cells via the cellular membrane. To explore the ability of the HLZ-BTED dots to diagnose breast cancer early, a mouse model of breast cancer was established. $4 \mathrm{~T} 1$ breast cancer cells (roughly $2 \times 10^{6}$ ) were subcutaneously injected into the right leg of female BALB/c mice. NIR-II fluorescence imaging of $4 \mathrm{~T} 1$ breast tumors was carried out by intravenously injecting the AIE dots $\left(0.2 \mathrm{~mL}, 10 \mathrm{mg} \mathrm{kg}^{-1}\right)$ into 4 T1 tumor-bearing mice $(n=3)$ through the tail vein when the tumors reached $\sim 60 \mathrm{~mm}^{3}$ in a week. The fluorescence intensity (1000 $\mathrm{nm} \mathrm{LP}, 100 \mathrm{~ms}$ ) in tumors reached the highest level at 48 h post-injection under $808 \mathrm{~nm}$ excitation with a power density of $90 \mathrm{~mW} \mathrm{~cm} \mathrm{~cm}^{-2}$ (Fig. 5 and $\mathrm{S} 8 \dagger$ ). For up to 8 days post-injection, the NIR-II fluorescence signal of tumors was extremely strong and clearly delineated the normal tissues, indicating that the AIE HLZ-BTED dots are adequate for long-term tumor imaging and real-time image-based monitoring for solid tumor therapy. An ex vivo biodistribution study was performed for the HLZ-BTED dots to evaluate their distribution in major organs at $192 \mathrm{~h}$ (Fig. S9†). High accumulation was observed in the liver
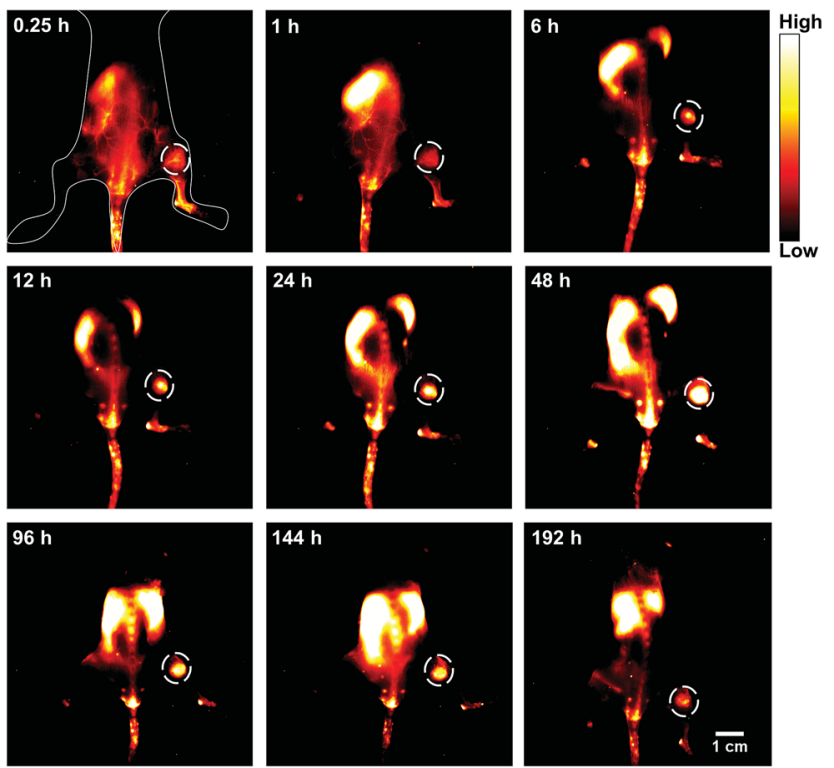

Fig. 5 Representative in vivo long-term NIR-II fluorescence images (808 nm excitation, $90 \mathrm{~mW} \mathrm{~cm}^{-2}, 1000 \mathrm{~nm} \mathrm{LP}, 100 \mathrm{~ms}, n=3$ ) of the $4 \mathrm{~T} 1$ breast tumor model at different time points after tail vein injection of the HLZ-BTED dots $\left(0.2 \mathrm{~mL}, 10 \mathrm{mg} \mathrm{kg}^{-1}\right)$. The white circles indicate the location of the $4 \mathrm{~T} 1$ tumor. Scale bar: $1 \mathrm{~cm}$

and spleen, which suggested that the clearance routes of the HLZ-BTED dots were through the hepatobiliary system. In addition, the AIE dots showed high accumulation in 4T1 tumors for up to 8 days (Fig. S9†).

Transcatheter arterial embolization (TAE) is widely used in the clinic to increase survival for cancer patients who are not suitable for chemotherapy and surgical resection. ${ }^{21}$ To estimate the potential feasibility of the HLZ-BTED dots as a NIR-II contrast agent for the in vivo imaging of the tumor-feeding blood vessels to implement TAE, the AIE HLZ-BTED dots were i.v. injected into $4 \mathrm{~T} 1$ tumor bearing BALB/c mice $(n=3)$. At 2 min post-injection, the tumor vasculature was visualized using an InGaAs camera with $1000 \mathrm{~nm}$ (Fig. 6A, $200 \mathrm{~ms}$ exposure time) and $1250 \mathrm{~nm}$ (Fig. 6B, $800 \mathrm{~ms}$ exposure time) long-pass filters under $808 \mathrm{~nm}$ laser excitation. The NIR-II fluorescence images of the 4T1 tumor blood vessels showed a more distinct vascular network with the $1250 \mathrm{~nm}$ long-pass filter than with the $1000 \mathrm{~nm}$ one, owing to both reduced biological auto-fluorescence and photon scattering in the longer wavelength NIR-II region. Meanwhile a similar image quality via different longpass filters has been demonstrated in the hind limb vasculature of C57BL/6 mice (Fig. 6C and D). Moreover, we performed a surgery to induce incomplete hind limb ischemia to mimic the NIR-II fluorescence imaging-guided vascular embolization procedure. The occlusion site of the blood supply vessels was precisely monitored by NIR-II imaging using the HLZ-BTED dots (Fig. 6E). Then the capability of long-term vasculature imaging using the HLZ-BTED dots was investigated. As shown in Fig. $6 \mathrm{~F}$, at $4 \mathrm{~h}$ post-injection, the vasculature was still clearly visualized through NIR-II imaging which would be adequate for surgical operation. The resolution $(497.6 \mu \mathrm{m})$ of the hind limb 
A)

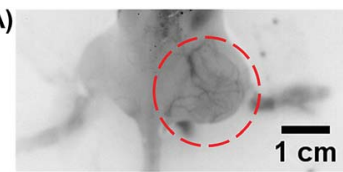

C)
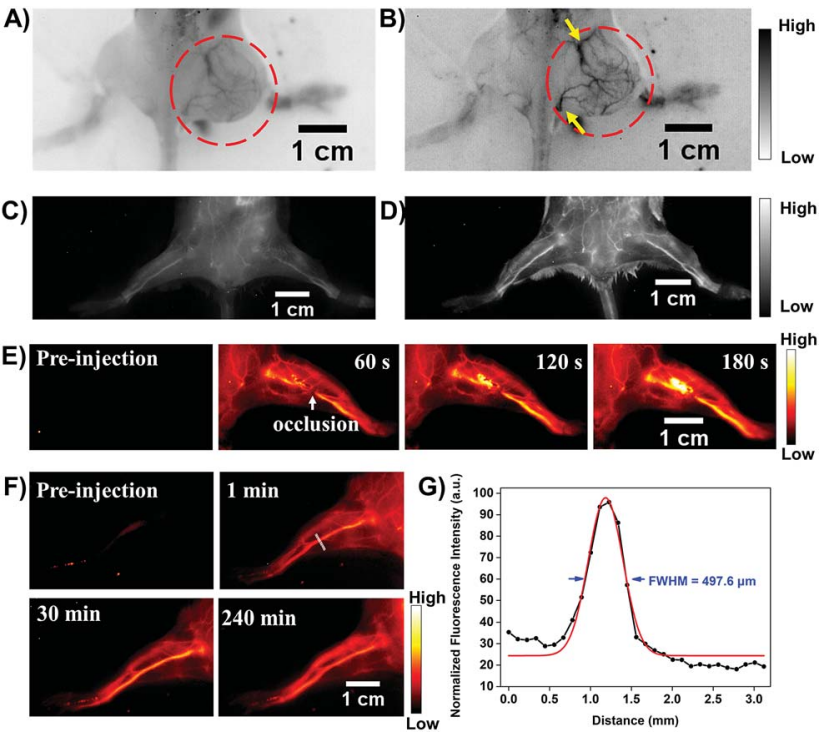

Fig. 6 (A and B) In vivo visualization of tumor-feeding vessels. NIR-II tumor blood vessel fluorescence images of the 4T1 breast tumorbearing mice were obtained at $2 \mathrm{~min}$ post tail vein injection of the HLZ-BTED dots $\left(0.2 \mathrm{~mL}, 10 \mathrm{mg} \mathrm{kg}^{-1}\right)$ with $1000 \mathrm{~nm}((\mathrm{~A}), 200 \mathrm{~ms}, 808$ $\mathrm{nm}$ excitation, $\left.90 \mathrm{~mW} \mathrm{~cm}^{-2}\right)$ and $1250 \mathrm{~nm}((B), 800 \mathrm{~ms}, 808 \mathrm{~nm}$ excitation, $180 \mathrm{~mW} \mathrm{~cm}^{-2}$ ) long-pass filters, respectively. The red circles indicate the location of the tumor, and yellow arrows indicate the tumor-feeding arteries. (C and D) NIR-II fluorescence images of the hind limb vessels in $\mathrm{C} 57 \mathrm{BL} / 6$ mice at 2 min after tail vein injection of the HLZ-BTED dots $\left(0.2 \mathrm{~mL}, 15 \mathrm{mg} \mathrm{kg}^{-1}\right)$ with $1000 \mathrm{~nm}$ ((C), $60 \mathrm{~ms}$, $808 \mathrm{~nm}$ excitation, $90 \mathrm{~mW} \mathrm{~cm}^{-2}$ ) and $1250 \mathrm{~nm}$ ((D), $500 \mathrm{~ms}, 808 \mathrm{~nm}$ excitation, $180 \mathrm{~mW} \mathrm{~cm}^{-2}$ ) long-pass filters, respectively. (E) In vivo NIR-II fluorescence images (1250 nm LP, 400 ms, 808 nm excitation, $180 \mathrm{~mW} \mathrm{~cm}^{-2}$ ) of incomplete left hind limb ischemia pre-injection and $60 \mathrm{~s}, 120 \mathrm{~s}$, and $180 \mathrm{~s}$ after HLZ-BTED dot injection, where the white arrow located the occlusion site in the femoral artery. (F) Intravital long-term hind limb vasculature NIR-II imaging (1250 nm LP, 400 ms, $808 \mathrm{~nm}$ excitation, $180 \mathrm{~mW} \mathrm{~cm}^{-2}$ ) from $0 \mathrm{~min}$ to $240 \mathrm{~min}$ after tail vein injection of the HLZ-BTED dots. Scale bar $(A-F): 1 \mathrm{~cm}$. (G) The vessel FWHM width based on the cross-sectional intensity profile measured along the white line in (F) (1 min) with the peak fitted to Gaussian functions (the red curve is the Gaussian fit to the profile).

vasculature was investigated via the Gaussian-fitted full width at half maximum (FWHM), which further confirmed the excellent ability of the HLZ-BTED dots to precisely map vascular vessels with NIR-II imaging (Fig. 6G).

The gastrointestinal (GI) tract is an organ system, which includes all organ structures between the mouth and the anus, such as the esophagus, stomach, small intestine, and large intestine. The GI tract is responsible for ingesting and digesting food, extracting and absorbing nutrients, and expelling the waste as feces. Recently, non-invasive imaging modalities such as positron emission tomography (PET), ${ }^{22}$ magnetic resonance imaging (MRI), ${ }^{23}$ X-ray computed tomography (CT), ${ }^{24}$ and photoacoustic (PA $)^{22}$ imaging, have played an important role in GI function research and the diagnosis and prognosis of GI diseases. However, these imaging modalities are limited by their serious drawbacks, notably radiation risk, expensive instrument cost, long imaging times and limited spatial resolution for real-time gut function study such as physiological or pathological intestinal motility. NIR-II fluorescence imaging may provide a reliable method for monitoring intestinal motility disorders or dysfunction and providing evaluation information of GI therapeutic agents in vivo due to superior temporal-spatial resolution. ${ }^{25}$ Therefore, as a proof of concept, we demonstrated the feasibility of the AIE HLZ-BTED dots for in vivo NIR-II imaging of the GI tract by oral administration. First, the fluorescence intensity and hydrodynamic sizes of the HLZ-BTED dots in different $\mathrm{pH}$ buffers ranging from 1 to 9 or in gastric pH 1 buffer were investigated respectively. No significant difference was observed between different conditions, demonstrating the excellent stability and capability of the AIE dots as a GI tract contrast agent (Fig. S10 $\dagger$ ). Second, healthy BALB/c mice $(n=3)$ were gavaged with the HLZ-BTED dots $(0.1 \mathrm{~mL}$, $5 \mathrm{mg} \mathrm{kg}^{-1}$ ) for real-time imaging of intestinal motility and monitoring the behavior of micelles within the GI tract. NIR-II fluorescence images at different time points $(5,10,20,30,60$, 90, 180, 480, 600 and $1440 \mathrm{~min}$ ) were obtained under $808 \mathrm{~nm}$ laser irradiation $\left(180 \mathrm{~mW} \mathrm{~cm} \mathrm{~cm}^{-2}\right.$, within the safety limits (329 $\mathrm{mW} \mathrm{cm}^{-2}$ ) determined by the International Commission on Non-ionizing Radiation Protection) through an InGaAs camera (200 ms exposure time) with a $1250 \mathrm{~nm}$ long-pass filter. As shown in Fig. 7A, from $5 \mathrm{~min}$ to $480 \mathrm{~min}$ after gavaging, the fluorescent signals were observed in the stomach, duodenum, jejunum, ileum, cecum, and colon. During the imaging procedure of the intestine, the contractile function of the intestine was demonstrated by the recorded Movie S1. $\uparrow$ The fluorescence intensity movement in the small intestine indicated vigorous

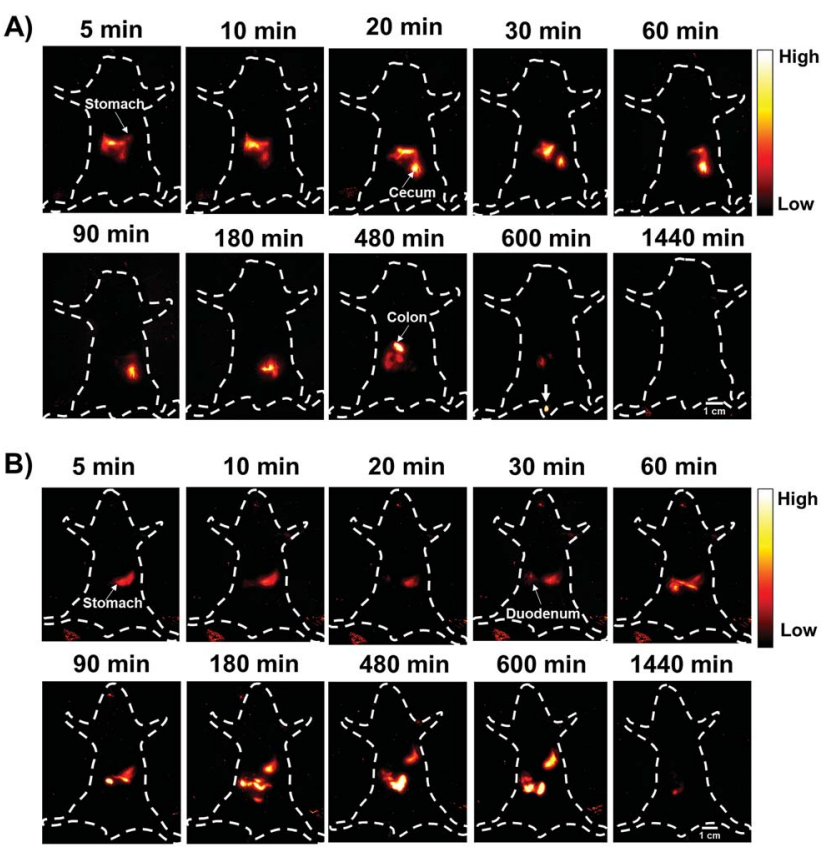

Fig. 7 ( $A$ and $B$ ) Representative non-invasive NIR-II fluorescence images (1250 nm LP, $200 \mathrm{~ms}, 808 \mathrm{~nm}$ excitation, $180 \mathrm{~mW} \mathrm{~cm}^{-2}$ ) of the gastrointestinal tract ( $\mathrm{Gl}$ tract) in BALB/c mice gavaged with the HLZBTED dots $\left(0.1 \mathrm{~mL}, 5 \mathrm{mg} \mathrm{kg}^{-1}\right)$ for real-time monitoring of gastrointestinal peristalsis of normal mice $(A)$ and mice anesthetized $(B)$ using pentobarbital sodium ( $n=3$ mice). The white arrow indicates the feces of mice (Fig. 7A, $600 \mathrm{~min}$ ). Scale bar: $1 \mathrm{~cm}$. 
contractility with peristalsis and segmental contractions of the intestine at $330 \mathrm{~min}$ after gavaging. By fluorescence intensity map analysis of regions of interest (ROIs), ${ }^{25 f}$ the intestine peristalsis frequency and propagation velocity was about 28-40 cycles $\mathrm{min}^{-1}$ and $2.61 \pm 0.23 \mathrm{~cm} \mathrm{~s}^{-1}$, respectively. At $600 \mathrm{~min}$ after gavaging, the fluorescent signal was first detected in feces, indicating that the NIR-II dots moved out of the body from the rectum and anus. At $24 \mathrm{~h}$ after gavaging, no fluorescent signal of the abdomen was detected. These results illustrated that the HLZ-BTED dots can be totally excreted through the GI tract within $24 \mathrm{~h}$ and are not absorbed into the body through the intestine. The HLZ-BTED dots used in GI tract imaging are excreted quickly due to low absorption in the GI tract while the HLZ-BTED dots would interact with serum plasma and the immune system by i.v. injection, leading to the slow clearance routes of the HLZ-BTED dots through the hepatobiliary system. Moreover, in Fig. 7B and 8C, the experimental results showed that the use of an anesthetic, pentobarbital sodium, could suppress duodenum motility and gastric emptying, indicating that the NIR-II HLZ-BTED dots could be a powerful tool for assessing the effects of drugs on the GI tract.

To investigate the ability of the HLZ-BTED dots to diagnose intestinal diseases such as obstructions which impede or block peristaltic movement, we built a mouse model of intestinal partial obstruction according to the reported methods. ${ }^{26}$ Then, we gavaged the mice with intestinal obstruction with the HLZBTED dots $\left(0.1 \mathrm{~mL}, 5 \mathrm{mg} \mathrm{kg}{ }^{-1}\right)$. The NIR-II images shown in Fig. 8A and 7A exhibited differences in the intestinal distribution of dots between the mice with obstruction and healthy mice. At $5 \mathrm{~h}$ after gavaging, the lower small intestine was fully filled and distended after dissection, and the fluorescent signal was strong in the upper and medial small intestine (Fig. 8B), indicating that the HLZ-BTED dots were accumulated in these parts and are suitable for diagnosing intestinal obstruction.

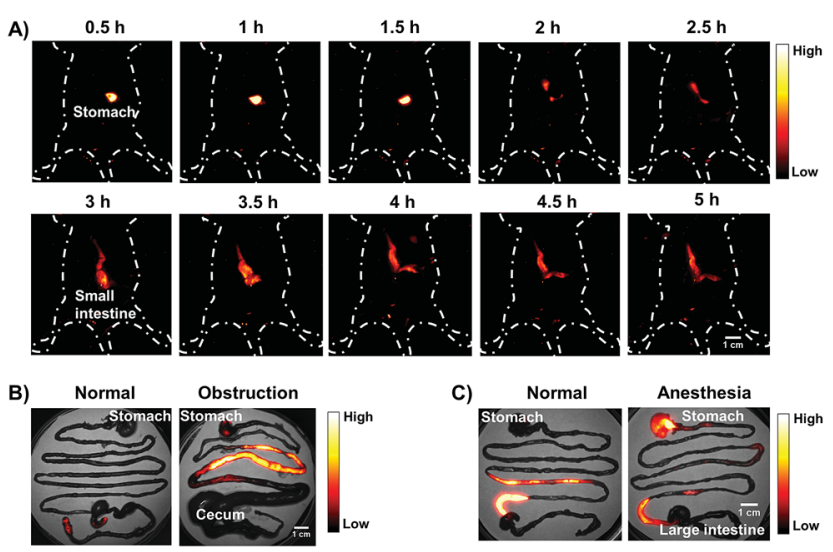

Fig. 8 (A) Representative non-invasive NIR-II fluorescence images (1250 nm LP, $200 \mathrm{~ms}, 808 \mathrm{~nm}$ excitation, $180 \mathrm{~mW} \mathrm{~cm}^{-2}$ ) of the gastrointestinal tract in BALB/c mice gavaged with the HLZ-BTED dots $\left(0.1 \mathrm{~mL}, 5 \mathrm{mg} \mathrm{kg}^{-1}\right.$ ) for visualizing intestinal obstruction ( $n=3$ mice). (B) Ex vivo NIR-II image (200 ms, $1250 \mathrm{~nm} \mathrm{LP})$ of the Gl tract in normal mice or mice with intestinal obstruction at $5 \mathrm{~h}$ after gavage. (C) Representative ex vivo NIR-II fluorescence image (1250 nm LP. $200 \mathrm{~ms}$ ) of the $\mathrm{Gl}$ tract in BALB/c mice which are normal or anesthetized by pentobarbital sodium at $2 \mathrm{~h}$ after gavage. Scale bar: $1 \mathrm{~cm}$.

\section{Experimental section}

All animal experiments were performed according to the Chinese Regulations for the Administration of Affairs Concerning Experimental Animals and approved by the Institutional Animal Care and Use Committee (IACUC) of Wuhan University. And all experimental details are provided in the ESI. $\dagger$

\section{Conclusions}

In conclusion, we have successfully designed and synthesized a novel NIR-II organic fluorophore HLZ-BTED with AIE features. The organic fluorophore could be encapsulated as HLZ-BTED dots with good water-solubility and biocompatibility for in vivo NIR-II biomedical imaging. We further performed in vivo NIR-II imaging experiments that revealed the excellent passive targeted long-term NIR-II fluorescence imaging performance of the HLZ-BTED dots in breast tumor-bearing mice. Moreover, the HLZ-BTED dots were an excellent NIR-II imaging contrast agent for superior spatial-temporal resolution of tumor-feeding vessels imaged with a $1250 \mathrm{~nm}$ long-pass filter, precisely monitoring the occlusion site of incomplete hind limb ischemia and long-term hind limb vasculature, and have the potential capability for image-guided transcatheter arterial embolization. To the best of our knowledge, as a proof-of-concept, this is the first time that non-invasive and real-time NIR-II imaging of the gastrointestinal tract in health and in disease has been performed. Furthermore, dynamic NIR-II fluorescence imaging with gavage of the HLZ-BTED dots can be used to monitor intestinal motility in real-time, and thus provides a method for the diagnosis of intestinal motility disorders and information on the response of the intestine to therapeutic agents.

\section{Conflicts of interest}

There are no conflicts to declare.

\section{Acknowledgements}

This work was partially supported by grants from the NSFC (81773674, 81573383, and 21390402), NSFHP (2017CFA024, 2017CFB711, and 2016ACA126), ABRPSTCS (SYG201521), NSFJP (BK20160387), Shenzhen Science and Technology Research Grant (JCYJ20170303170809222), Fundamental Research Funds for the Central Universities and Open Research Fund Program of the Hubei Province Engineering and Technology Research Center for Fluorinated Pharmaceuticals.

\section{Notes and references}

1 (a) X. D. Zhang, H. Wang, A. L. Antaris, L. Li, S. Diao, R. Ma, A. Nguyen, G. Hong, Z. Ma, J. Wang, S. Zhu, J. M. Castellano, T. Coray, Y. Liang, J. Luo and H. Dai, Adv. Mater., 2016, 28, 6872-6879; (b) H. Wan, J. Yue, S. Zhu, T. Uno, X. Zhang, Q. Yang, K. Yu, G. Hong, J. Wang, L. Li, Z. Ma, H. Gao, 
Y. Zhong, J. Su, A. L. Antaris, Y. Xia, J. Luo, Y. Liang and H. Dai, Nat. Commun., 2018, 9, 1171.

2 (a) E. C. Rossi, A. Ivanova and J. F. Boggess, Gynecol. Oncol., 2012, 124, 78-82; (b) G. Wishart, S.-W. Loh, L. Jones and J. Benson, Eur. J. Surg. Oncol., 2012, 38, 651-656; (c) C. Hirche, D. Murawa, Z. Mohr, S. Kneif and M. Hünerbein, Breast Cancer Res. Treat., 2010, 121, 373-378.

3 (a) M. Herranz and A. Ruibal, J. Oncol., 2012, 2012, 863747; (b) C. Zeng, W. Shang, X. Liang, X. Liang, Q. Chen, C. Chi, Y. Du, C. Fang and J. Tian, ACS Appl. Mater. Interfaces, 2016, 8, 29232-29241; (c) S. Luo, E. Zhang, Y. Su, T. Cheng and C. Shi, Biomaterials, 2011, 32, 7127-7138; (d) J. Yang, Q. Xie, H. Zhou, L. Chang, W. Wei, Y. Wang, H. Li, Z. Deng, Y. Xiao, J. Wu, P. $\mathrm{Xu}$ and $\mathrm{X}$. Hong, J. Proteome Res., 2018, 17, 2428-2439.

4 (a) Y. Sun, X. Zeng, Y. Xiao, C. Liu, H. Zhu, H. Zhou, Z. Chen, F. Xu, J. Wang, M. Zhu, J. Wu, M. Tian, H. Zhang, Z. Deng, Z. Cheng and X. Hong, Chem. Sci., 2018, 9, 2092-2097; (b) Y. Sun, M. Ding, X. Zeng, Y. Xiao, H. Wu, H. Zhou, B. Ding, C. Qu, W. Hou, A. Erbu, Y. Zhang, Z. Cheng and X. Hong, Chem. Sci., 2017, 8, 3489-3493.

5 (a) F. Ding, Y. Zhan, X. Lu and Y. Sun, Chem. Sci., 2018, 9, 4370-4380; (b) G. Hong, A. L. Antaris and H. Dai, Nat. Biomed. Eng., 2017, 1, 0010; (c) F. Ding, S. Chen, W. Zhang, Y. Tu and Y. Sun, Bioorg. Med. Chem., 2017, 25, 5179-5184; (d) Y. Xu, M. Tian, H. Zhang, Y. Xiao, X. Hong and Y. Sun, Chin. Chem. Lett., 2018, 29, 1093-1097; (e) S. He, J. Song, J. Qu and Z. Cheng, Chem. Soc. Rev., 2018, 47, 4258-4278; (f) W. Zhu, Sci. China: Chem., 2016, 59, 203-204; (g) B. Li, L. Lu, M. Zhao, Z. Lei and F. Zhang, Angew. Chem., Int. Ed., 2018, 57, 7483-7487; (h) H. Zhou, Y. Xiao and X. Hong, Chin. Chem. Lett., 2018, 29, 1425-1428; (i) J. Yang and X. Hong, Sci. China: Chem., 2018, DOI: 10.1007/s11426-0189341-7.

6 (a) L. Liu, S. Wang, B. Zhao, P. Pei, Y. Fan, X. Li and F. Zhang, Angew. Chem., 2018, 130, 7640-7644; (b) Y. Fan, P. Wang, Y. Lu, R. Wang, L. Zhou, X. Zheng, X. Li, J. A. Piper and F. Zhang, Nat. Nanotechnol., 2018, 13, 941-946; (c) P. Wang, Y. Fan, L. Lu, L. Liu, L. Fan, M. Zhao, Y. Xie, C. Xu and F. Zhang, Nat. Commun., 2018, 9, 2898; (d) Y. Zhong, Z. Ma, S. Zhu, J. Yue, M. Zhang, A. L. Antaris, J. Yuan, R. Cui, H. Wan, Y. Zhou, W. Wang, N. F. Huang, J. Luo, Z. Hu and H. Dai, Nat. Commun., 2017, 8, 737; (e) X. Jiang, C. Cao, W. Feng and F. Li, J. Mater. Chem. B, 2016, 4, 87-95; $(f)$ D. Naczynski, M. Tan, M. Zevon, B. Wall, J. Kohl, A. Kulesa, S. Chen, C. Roth, R. Riman and P. Moghe, Nat. Commun., 2013, 4, 2199.

7 (a) M. Zhang, J. Yue, R. Cui, Z. Ma, H. Wan, F. Wang, S. Zhu, Y. Zhou, Y. Kuang, Y. Zhong, D.-W. Pang and H. Dai, Proc. Natl. Acad. Sci. U. S. A., 2018, 201806153; (b) Z. Ma, M. Zhang, J. Yue, C. Alcazar, Y. Zhong, T. C. Doyle, H. Dai and N. F. Huang, Adv. Funct. Mater., 2018, 28, 1803417; (c) G. Chen, S. Lin, D. Huang, Y. Zhang, C. Li, M. Wang and Q. Wang, Small, 2018, 14, 1702679; (d) C. Li, Y. Zhang, G. Chen, F. Hu, K. Zhao and Q. Wang, Adv. Mater., 2017, 29, 1605754; (e) G. Chen, F. Tian, C. Li, Y. Zhang, Z. Weng, Y. Zhang, R. Peng and Q. Wang, Biomaterials, 2015, 53,
265-273; $(f)$ G. Hong, J. T. Robinson, Y. Zhang, S. Diao, A. L. Antaris, Q. Wang and H. Dai, Angew. Chem., Int. Ed., 2012, 51, 9818-9821; $(g)$ Y. Zhang, G. Hong, Y. Zhang, G. Chen, F. Li, H. Dai and Q. Wang, ACS Nano, 2012, 6, 3695-3702.

8 (a) G. Hong, S. Diao, J. Chang, A. L. Antaris, C. Chen, Z. Bo, Z. Su, D. N. Atochin, P. L. Huang, K. I. Andreasson, C. J. Kuo and H. Dai, Nat. Photonics, 2014, 8, 723-730; (b) J. T. Robinson, K. Welsher, S. M. Tabakman, S. P. Sherlock, H. Wang, R. Luong and H. Dai, Nano Res., 2010, 3, 779-793. 9 (a) K. Shou, Y. Tang, H. Chen, S. Chen, L. Zhang, A. Zhang, Q. Fan, A. Yu and Z. Cheng, Chem. Sci., 2018, 9, 3105-3110; (b) G. Hong, Y. Zou, A. L. Antaris, S. Diao, D. Wu, K. Cheng, X. Zhang, C. Chen, B. Liu, Y. He, J. Z. Wu, J. Yuan, B. Zhang, Z. Tao, C. Fukunaga and H. Dai, Nat. Commun., 2014, 5, 4206.

10 (a) A. L. Antaris, H. Chen, K. Cheng, Y. Sun, G. Hong, C. Qu, S. Diao, Z. Deng, X. Hu, B. Zhang, X. Zhang, O. K. Yaghi, Z. R. Alamparambil, X. Hong, Z. Cheng and H. Dai, Nat. Mater., 2016, 15, 235-242; (b) A. L. Antaris, H. Chen, S. Diao, Z. Ma, Z. Zhang, S. Zhu, J. Wang, A. X. Lozano, Q. Fan, L. Chew, M. Zhu, K. Cheng, X. Hong, H. Dai and Z. Cheng, Nat. Commun., 2017, 8, 15269; (c) Y. Feng, S. Zhu, A. L. Antaris, H. Chen, Y. Xiao, X. Lu, L. Jiang, S. Diao, K. Yu, Y. Wang, S. Herraiz, J. Yue, X. Hong, G. Hong, Z. Cheng, H. Dai and A. J. Hsueh, Chem. Sci., 2017, 8, 3703-3711.

11 (a) K. Shou, C. Qu, Y. Sun, H. Chen, S. Chen, L. Zhang, H. Xu, X. Hong, A. Yu and Z. Cheng, Adv. Funct. Mater., 2017, 27, 1700995; (b) S. Zhu, Q. Yang, A. L. Antaris, J. Yue, Z. Ma, H. Wang, W. Huang, H. Wan, J. Wang, S. Diao, B. Zhang, X. Li, Y. Zhong, K. Yu, G. Hong, J. Luo, Y. Liang and H. Dai, Proc. Natl. Acad. Sci. U. S. A., 2017, 114, 962-967; (c) Q. Yang, Z. Ma, H. Wang, B. Zhou, S. Zhu, Y. Zhong, J. Wang, W. Hao, A. Antaris, M. Rui, X. Zhang, J. Yang, X. Zhang, H. Sun, W. Liu, Y. Liang and H. Dai, Adv. Mater., 2017, 29, 1605497; (d) Y. Sun, C. Qu, H. Chen, M. He, C. Tang, K. Shou, S. Hong, M. Yang, Y. Jiang, B. Ding, Y. Xiao, L. Xing, X. Hong and Z. Cheng, Chem. Sci., 2016, 7, 6203-6207; (e) Q. Yang, Z. Hu, S. Zhu, R. Ma, H. Ma, Z. Ma, H. Wan, T. Zhu, Z. Jiang, W. Liu, L. Jiao, H. Sun, Y. Liang and H. Dai, J. Am. Chem. Soc., 2018, 140, 17151724; $(f)$ S. Zhu, S. Herraiz, J. Yue, M. Zhang, H. Wan, Q. Yang, Z. Ma, Y. Wang, J. He, A. L. Antaris, Y. Zhong, S. Diao, Y. Feng, Y. Zhou, K. Yu, G. Hong, Y. Liang, A. J. Hsueh and H. Dai, Adv. Mater., 2018, 30, 1705799.

12 J. Luo, Z. Xie, J. W. Lam, L. Cheng, H. Chen, C. Qiu, H. S. Kwok, X. Zhan, Y. Liu, D. Zhu and B. Z. Tang, Chem. Commun., 2001, 1740-1741.

13 (a) Z. Chen, J. Zhang, M. Song, J. Yin, G.-A. Yu and S. H. Liu, Chem. Commun., 2015, 51, 326-329; (b) J. Mei, N. L. Leung, R. T. Kwok, J. W. Lam and B. Z. Tang, Chem. Rev., 2015, 115, 11718-11940; (c) G. Feng and B. Liu, Acc. Chem. Res., 2018, 51, 1404-1414; (d) J. Shi, Q. Deng, C. Wan, M. Zheng, F. Huang and B. Tang, Chem. Sci., 2017, 8, 6188-6195; (e) T. He, N. Niu, Z. Chen, S. Li, S. Liu and J. Li, Adv. Funct. Mater., 2018, 28, 1706196; (f) Q. Hu, M. Gao, G. Feng and 
B. Liu, Angew. Chem., Int. Ed., 2014, 53, 14225-14229; (g)

S. Zhen, S. Wang, S. Li, W. Luo, M. Gao, L. G. Ng, C. C. Goh, A. Qin, Z. Zhao and B. Liu, Adv. Funct. Mater., 2018, 28, 1706945.

14 J. Qi, C. Sun, A. Zebibula, H. Zhang, R. T. Kwok, X. Zhao, W. Xi, J. W. Lam, J. Qian and B. Z. Tang, Adv. Mater., 2018, 30, 1706856.

15 Z. Sheng, B. Guo, D. Hu, S. Xu, W. Wu, W. H. Liew, K. Yao, J. Jiang, C. Liu, H. Zheng and B. Liu, Adv. Mater., 2018, 30, 1800766.

16 (a) F. Zhao, Z. Chen, G. Liu, C. Fan and S. Pu, Tetrahedron Lett., 2018, 59, 836-840; (b) B. Xu, J. He, Y. Mu, Q. Zhu, S. Wu, Y. Wang, Y. Zhang, C. Jin, C. Lo, Z. Chi, A. Lien, S. Liua and J. Xu, Chem. Sci., 2015, 6, 3236-3241; (c) Z. Zhang, R. T. Kwok, Y. Yu, B. Z. Tang and K. M. Ng, J. Mater. Chem. B, 2018, 6, 4575-4578; (d) J. Mei, Y. Hong, J. W. Lam, A. Qin, Y. Tang and B. Z. Tang, Adv. Mater., 2014, 26, 5429-5479; (e) S. Xu, Y. Yuan, X. Cai, C.-J. Zhang, F. Hu, J. Liang, G. Zhang, D. Zhang and B. Liu, Chem. Sci., 2015, 6, 5824-5830; (f) Y. Yuan, C. J. Zhang, M. Gao, R. Zhang, B. Z. Tang and B. Liu, Angew. Chem., Int. Ed., 2015, 54, 1780-1786.

17 (a) H. Shi, S. Wang, L. Fang and B. Z. Tang, Tetrahedron Lett., 2016, 57, 4428-4434; (b) X. Y. Shen, Y. J. Wang, E. Zhao, W. Z. Yuan, Y. Liu, P. Lu, A. Qin, Y. Ma, J. Z. Sun and B. Z. Tang, J. Phys. Chem. C, 2013, 117, 7334-7347.

18 X. Zeng, Y. Xiao, J. Lin, S. Li, H. Zhou, J. Nong, G. Xu, H. Wang, F. Xu, J. Wu, Z. Deng and X. Hong, Adv. Healthcare Mater., 2018, 7, 1800589.

19 (a) J. Ferlay, I. Soerjomataram, R. Dikshit, S. Eser, C. Mathers, M. Rebelo, D. M. Parkin, D. Forman and F. Bray, Int. J. Cancer, 2015, 136, E359-E386; (b) M. Ding, H. Wang, C. Qu and X. Hong, Cancer Lett., 2018, 432, 4755; (c) C. Qu, M. Ding, H. Wang, M. X. Zhu and X. Hong, J. Med. Chem., 2017, 60(11), 4680-4692.

20 C. Burgess, V. Cornelius, S. L ove, J. Graham, M. Richards and A. Ramirez, BMJ, 2005, 330, 702.

21 Ş. Aksoy, B. Akçe, Ö. Kılıçkesmez, R. U. Gürsü, M. S. Çakır, M. A. Nazlı and A. Aren, Journal of Breast Health, 2016, 12, 137-140.
22 (a) Y. Zhang, M. Jeon, L. J. Rich, H. Hong, J. Geng, Y. Zhang, S. Shi, T. E. Barnhart, P. Alexandridis, J. D. Huizinga, M. Seshadri, W. Cai, C. Kim and J. F. Lovell, Nat. Nanotechnol., 2014, 9, 631-638; (b) Y. Zhang, D. Wang, S. Goel, B. Sun, U. Chitgupi, J. Geng, H. Sun, T. E. Barnhart, W. Cai, J. Xia and J. F. Lovell, Adv. Mater., 2016, 28, 8524-8530.

23 (a) J. Huang, Q. Shu, L. Wang, H. Wu, A. Y. Wang and H. Mao, Biomaterials, 2015, 39, 105-113; (b) V. S. Perera, G. Chen, Q. Cai and S. D. Huang, Analyst, 2016, 141, 20162022; (c) A. Khalaf, C. L. Hoad, R. C. Spiller, P. A. Gowland, G. W. Moran and L. Marciani, World J. Gastrointest. Pathophysiol., 2015, 6, 140-149; (d) J. Alyami, R. Spiller and L. Marciani, Neurogastroenterol. Motil., 2015, 27, 1687-1692. 24 (a) X. Zheng, J. Shi, Y. Bu, G. Tian, X. Zhang, W. Yin, B. Gao, Z. Yang, Z. Hu, X. Liu, L. Yan, Z. Gu and Y. Zhao, Nanoscale, 2015, 7, 12581-12591; (b) Z. Liu, X. Ran, J. Liu, Y. Du, J. Ren and X. Qu, Biomaterials, 2016, 100, 17-26; (c) Z. Liu, J. Liu, R. Wang, Y. Du, J. Ren and X. Qu, Biomaterials, 2015, 56, 206-218.

25 (a) M. G. Panthani, T. A. Khan, D. K. Reid, D. J. Hellebusch, M. R. Rasch, J. A. Maynard and B. A. Korgel, Nano Lett., 2013, 13, 4294-4298; (b) R. Wang, L. Zhou, W. Wang, X. Li and F. Zhang, Nat. Commun., 2017, 8, 14702; (c) N. GevaZatorsky, D. Alvarez, J. E. Hudak, N. C. Reading, D. ErturkHasdemir, S. Dasgupta, U. H. Von Andrian and D. L. Kasper, Nat. Med., 2015, 21, 1091-1100; (d) J. Li, S. Thamphiwatana, W. Liu, B. Esteban-Fernández de Ávila, P. Angsantikul, E. Sandraz, J. Wang, T. Xu, F. Soto, V. Ramez, X. Wang, W. Gao, L. Zhang and J. Wang, ACS Nano, 2016, 10, 9536-9542; (e) S. Kwon, C. Davies-Venn and E. M. Sevick-Muraca, Neurogastroenterol. Motil., 2012, 24, 494-497; $(f)$ S. Kwon and E. M. Sevick-Muraca, Neurogastroenterol. Motil., 2011, 23, 881; $(g)$ K. Tahara, M. Nishio and H. Takeuchi, Drug Dev. Ind. Pharm., 2018, 44, 608-614.

26 S. Ha, L. Wei, B. Jorgensen, M. Lee, P. Park, S. Poudrier and S. Ro, J. Visualized Exp., 2018, 133, e57381. 